\title{
Estilo de vida y su relación con el estado nutricional de los trabajadores del Instituto Politécnico de la Salud, UNAN-Managua
}

MSc. Jenny del Carmen Casco Palma

Departamento de Nutrición UNAN-MANAGUA, POLISAL jennycasco65@yahoo.es

DOI: https://doi.org/10.5377/torreon.v7i18.7716

Palabras clave: Estilos de vida, hábitos alimentarios, estado nutricional.

\section{RESUMEN}

位 de los trabajadores del Instituto Politécnico de Salud POLISAL, se realizó un estudio descriptivo, correlacional y analítico. Fueron analizados los datos sobre las características sociodemográficas, estado nutricional y estilos de vida, entre ellos; hábitos alimentarios, actividad y ejercicio físico, patrones de sueño y manejo de estrés y uso de sustancias tóxicas, como alcohol y tabaco. Los análisis estadísticos efectuados fueron: descriptivos, pruebas de Correlación no Paramétrica V de Cramer. Del análisis y discusión de los resultados obtenidos, se alcanzaron las siguientes conclusiones: el $86 \%$ presentó sobrepeso o algún grado de obesidad, situación que se agudiza en el 96 \% de los docentes. Un patrón alimentario compuesto de 10 alimentos: chiltoma, aceite, cebolla, tomate, arroz, azúcar, frijoles, café, pollo y pan, con una dieta poco diversa, su principal fuente de adquisición calórica son las grasas, el 74 \% no realiza ejercicio físico, el 67 \% tiene buen manejo del estrés, bajo consumo de alcohol y tabaco, no existe una relación de asociación significativa entre los estilos de vida y el estado nutricional con un $p=0.42$. 


\section{INTRODUCCIÓN}

La carga mundial de enfermedades crónicas no transmisibles sigue en aumento, poder frenar o disminuir esta situación es un reto para los gobiernos. Datos actuales revelan que en el 2005, estas enfermedades causaron alrededor de 35 millones de defunciones, principalmente muertes por enfermedades cardiovasculares, diabetes y cáncer, registrándose más en países de ingresos bajos y medianos (OMS, 2011). Diversos estudios han concluido que dicha situación se puede prevenir y que está estrechamente relacionada con la modificación de los estilos de vida, como uno de los factores de riesgo modificable. Los estilos de vida saludables catalogados, como el conjunto de conductas positivas que todo ser humano debe realizar para mantener un estado de salud que permita obtener el completo estado de bienestar físico, mental y social según refiere Elliot (citado en Pastor, 1998), y que entre estas conductas saludables se pueden citar el ejercicio físico, una adecuada alimentación, patrones de sueño adecuados, no abuso de sustancias nocivas.

En Nicaragua, los problemas de salud relacionados con los estilos de vida han ido en aumento en los últimos años, en donde el 9.7\% de la población padece de Enfermedades Crónicas No Transmisibles (ECNT) agrupándose el mayor número de casos en el área urbana y siendo la población no pobre la más afectada según refiere la Fundación Nicaragüense para el Desarrollo Económico y Social (FUNIDES,2012), esta situación ha provocado que en la actualidad dentro de las políticas de salud del estado se elaboren estrategias dirigidas hacia la prevención.

Partiendo de este enfoque, nace la preocupación de que en Nicaragua existen pocos estudios sobre ¿cómo son los estilos de vida de los diferentes grupos de población? y ¿cómo es el comportamiento de los trabajadores hacia el cuido de su salud? situación que también es desconocida en el ámbito universitario. Es importante que el Instituto Politécnico de la Salud- POLISAL, como unidad formadora de profesionales afines a la salud y como parte de la universidad saludable, identifique sus estilos de vida, puesto que a través de ello se podrá detectar oportunamente la influencia que estos tengan sobre el desarrollo de enfermedades crónicas no transmisibles que vienen a limitar el desempeño de sus funciones por el deterioro a su salud.

Este estudio tiene como fin evaluar los estilos de vida y su relación con el estado nutricional de los trabajadores del POLISAL. Esto va a permitir a su vez, elaborar estrategias, en el marco de las universidades saludables, que ayuden a la comunidad universitaria a la elección de estilos de vida que preserven su salud. 


\section{MATERIAL Y MÉTODO}

De acuerdo al método de investigación, el presente estudio es observacional, descriptivo de tipo correlacional. Según al tiempo de ocurrencia de los hechos y registro de la información es retro-prospectivo; por el período y secuencia es transversal y según el análisis y alcance de los resultados es analítico, bajo el enfoque mixto, con un diseño observacional no experimental con predominio en el enfoque cuantitativo. El área de estudio está constituida por el Instituto Politécnico de la Salud (POLISAL) Luis Felipe Moncada de la Universidad Nacional Autónoma de Nicaragua UNAN-Managua.

El universo está compuesto por los 77 trabajadores de planta del POLISAL, estos distribuidos dela siguiente manera: 46 docentes y 31 administrativos. Para los datos cuantitativos no se calculó muestra ni se realizó muestreo probabilístico. La muestra estuvo constituida por 57 trabajadores que quisieron formar parte del mismo a quienes se les administró una encuesta y a 5 a los que se les aplicó una guía de observación. Para los datos cualitativos se realizó una entrevista a 7 informantes claves como: tomadores de decisiones y conocedores del tema. El total de individuos incluidos en la presente investigación fue de 69 sujetos, que sí cumplieron los criterios de inclusión.

Para la recolección de los datos cuantitativos el instrumento utilizado fue un cuestionario y el método la encuesta, la cual estuvo compuesta de tres acápites: Características sociodemográficos, estado nutricional y de salud, que contempla los subacápites sobre datos antropométricos y antecedentes personales patológicos y el estilo de vida el cual se enfocó en identificar la actividad física, tipo de alimentación, consumo de tabaco y alcohol y horas sueño y manejo de estrés; con el objetivo de identificar los alimentos y preparaciones de mayor consumo se utilizó una guía de observación estructurada. Para determinar el patrón alimentario se utilizó una frecuencia de consumo de alimentos de siete días cuantificado con el propósito de estimar el aporte calórico de los diferentes grupos de alimentos y poder determinar su adecuación calórica. Los datos cualitativos se recopilaron a través de una entrevista semiestructurada que contiene cinco preguntas abiertas, siendo estas la guía para el desarrollo de la temática (Bracker, 1998).

A partir de los datos recolectados, se diseñó la base de datos correspondientes, utilizando el software estadístico SPSS, v. 20 para Windows. Una vez que se realizó el control de calidad de los datos registrados, fueron elaborados los análisis estadísticos adecuados a la variable de estudio. De acuerdo a la naturaleza de cada una de las variables cuantitativas para cada uno de los objetivos específicos se realizaron los análisis descriptivos correspondientes a las variables nominales, entre ellos; análisis de frecuencia, estadísticas descriptivas de tendencia central, para las variables no paramétricas se les realizó análisis de contingencia (crosstab análisis). Para el 
objetivo de asociación se realizó análisis de contingencia para las variables no paramétricas y se les aplicó la prueba de relación de asociación V de Cramer.

\section{RESULTADOS Y DISCUSIÓN DE LOS RESULTADOS}

En la investigación realizada se encontró que la edad promedio es de 38 años; de estos, el 82 \% reside en el casco urbano. Al evaluarse el estado nutricional a través del IMC, solo el $14 \%$ resultó encontrarse en estado normal, el 86 \% sufre de sobrepeso o algún grado de obesidad. Al ser evaluados por área de trabajo, el 96 \% de los docentes sufre de esta patología. Estos hallazgos se relacionan con la situación que se presenta a nivel del país en donde el 30 \% de la población adulta sufre esta misma condición, y de igual manera, residen en las zonas urbanas (OPS, 2006). Datos similares se demostraron en un estudio realizado por Aranceta et. al (2005) sobre la prevalencia de obesidad en España en el que el 54.7 \% de la población entre 25 y 64 años resultó presentar esta misma situación.

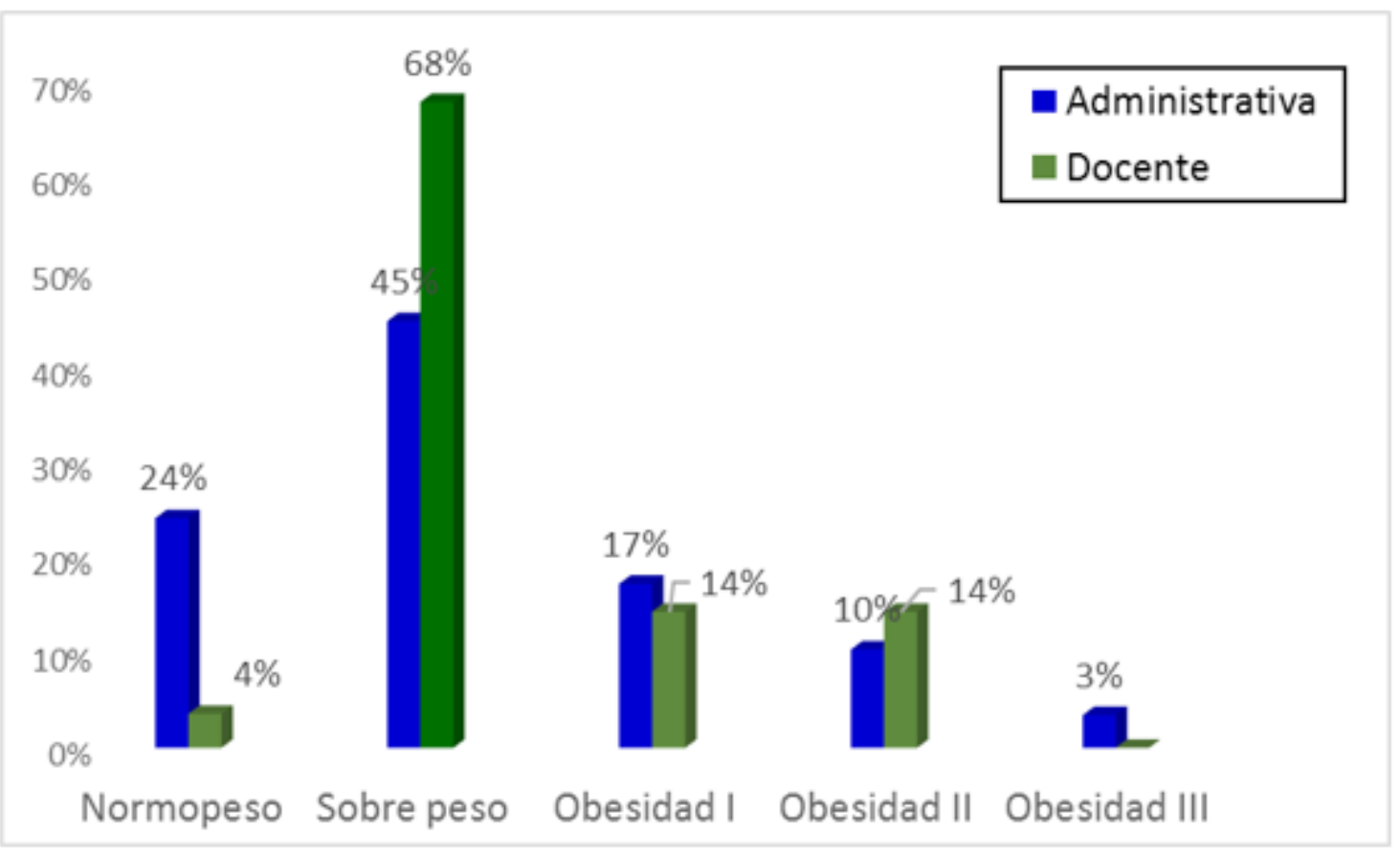

Figura 1. Índice de masa corporal de los trabajadores del POLISAL según área laboral

Palomares (2014) presenta la misma tendencia en el estudio en Lima, en trabajadores de la salud en el que el $68 \%$ de los profesionales presentaron sobrepeso u obesidad. Si se comparan estos tres estudios, se puede identificar que la tendencia hacia el incremento de peso es preocupante en el que más de la mitad de esta población se encuentra con uno de los factores de riesgo modificables para el desarrollo de enfermedades que pueden tener un curso irreversible hacia el daño a la salud.

Es importante señalar que, de los trabajadores que presentan un IMC por encima de 25, el 8.8 \% padece de una ECNT, entre ellas, diabetes e hipertensión. La FAO (2014) refiere 
que este problema es un factor desencadenante para el desarrollo de esta patología y que a nivel mundial, su edad de aparición es cada vez más en la población adulta joven, en este estudio la población con esta clasificación nutricional es mucho más elevada que las cifras a nivel nacional y en comparación con la investigación realizada por González (2009) en Barcelona.

$\mathrm{Al}$ analizar los hábitos alimentarios de los trabajadores, entre las prácticas inadecuadas destacan que el $88 \%$ nunca realiza los 5 o 6 tiempos de comida; el $84 \%$ siempre agrega sal a los alimentos; y el 95 \% nunca evita el consumo de refrescos industrializados. Sin embargo, entre las prácticas adecuadas se encontró que el 53 \% consume de 6 a 8 vasos de agua al día; el $61 \%$ trae la comida de su casa; y el 95 \% ingiere agua con las comidas. Igualmente, se pudo identificar a través del método de frecuencia de alimentos cuantificado, que el patrón alimentario de los trabajadores está compuesto solamente por 10 alimentos.

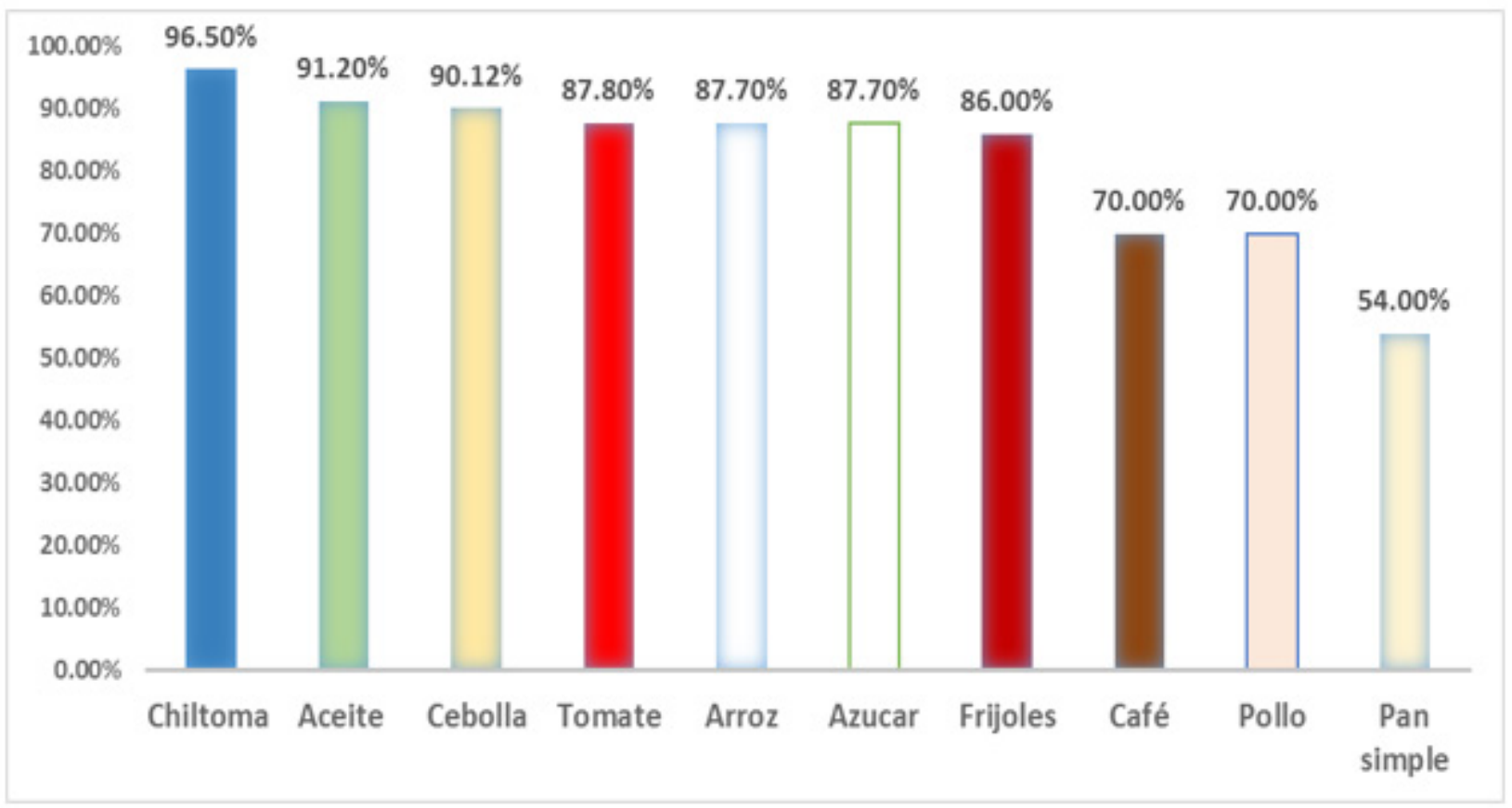

Figura 2. Patrón alimentario de los trabajadores del POLISAL

Esta figura muestra la dieta representada por cinco alimentos fuentes de carbohidratos, de los cuales, dos son carbohidratos sencillos (azúcar y panes), tres, son alimentos que proporcionan vitaminas y minerales y sin alimentos fuentes de fibra. Esta alimentación se encuentra con menos variedad en comparación al patrón a nivel nacional el cual lo componen 21 alimentos (FAO, 2007), mostrando un mayor déficit en la diversidad, lo que conlleva a que la distribución de la fuente calórica se reduzca a unos cuantos productos provocando así un desbalance energético.

En este sentido, el estudio de Ratnar et al (2008) en el que caracterizaron los hábitos alimentarios de trabajadores de empresas públicas y privadas de Chile, identificando un bajo consumo de frutas, verduras, pescado y productos lácteos y un alto consumo de grasa y azúcar. Al analizar la principal fuente de adquisición calórica, sobresalen las grasas, en la que más del 75 \% 
de la población oscila entre $38.8 \%$ y $55.5 \%$, la que proviene principalmente de las preparaciones fritas adquiridas en los diferentes bares de la universidad.

La segunda fuente calórica procede de las proteínas la cual oscila entre $29.7 \%$ y $47.9 \%$ derivada de la carne de cerdo, res y queso y el aporte que proviene de los carbohidratos se encuentra entre $11 \%$ y 15.8 \%, lo que evidencia que la distribución de sus fuentes de calorías no es la adecuada, puesto que esta tiene que ser principalmente procedente de los carbohidratos.

Dentro de los factores que inciden de manera directa sobre el estado nutricional es la actividad física, la cual, según refiere la OMS (2010) para los adultos debe ser realizada al menos 150 minutos semanales con una intensidad moderada y que para obtener mejores resultados se debe aumentar hasta 300 minutos para mantener un buen estado de salud. En la figura 3 se puede observar que el $74 \%$ de los trabajadores no practica ningún tipo de ejercicio programado.

Si se comparan estos resultados con los del estudio en profesionales de salud de Colombia realizado por Sanabria, González y Urrego (2007), al evaluar el nivel de actividad física identificaron que el 85.6 \% de los médicos y el 94.1 \% de las enfermeras presentaron hábitos poco y nada saludables. Se puede observar que esta actividad es cada día practicada con menos frecuencia, lo que viene a repercutir negativamente en el estado nutricional y de salud en general, porque, al aumentar esta actividad, se tiende a disminuir significativamente el peso, la presión arterial, la glicemia y mejora el estado general según datos de un estudio realizado en Chile por Salinas (2005).

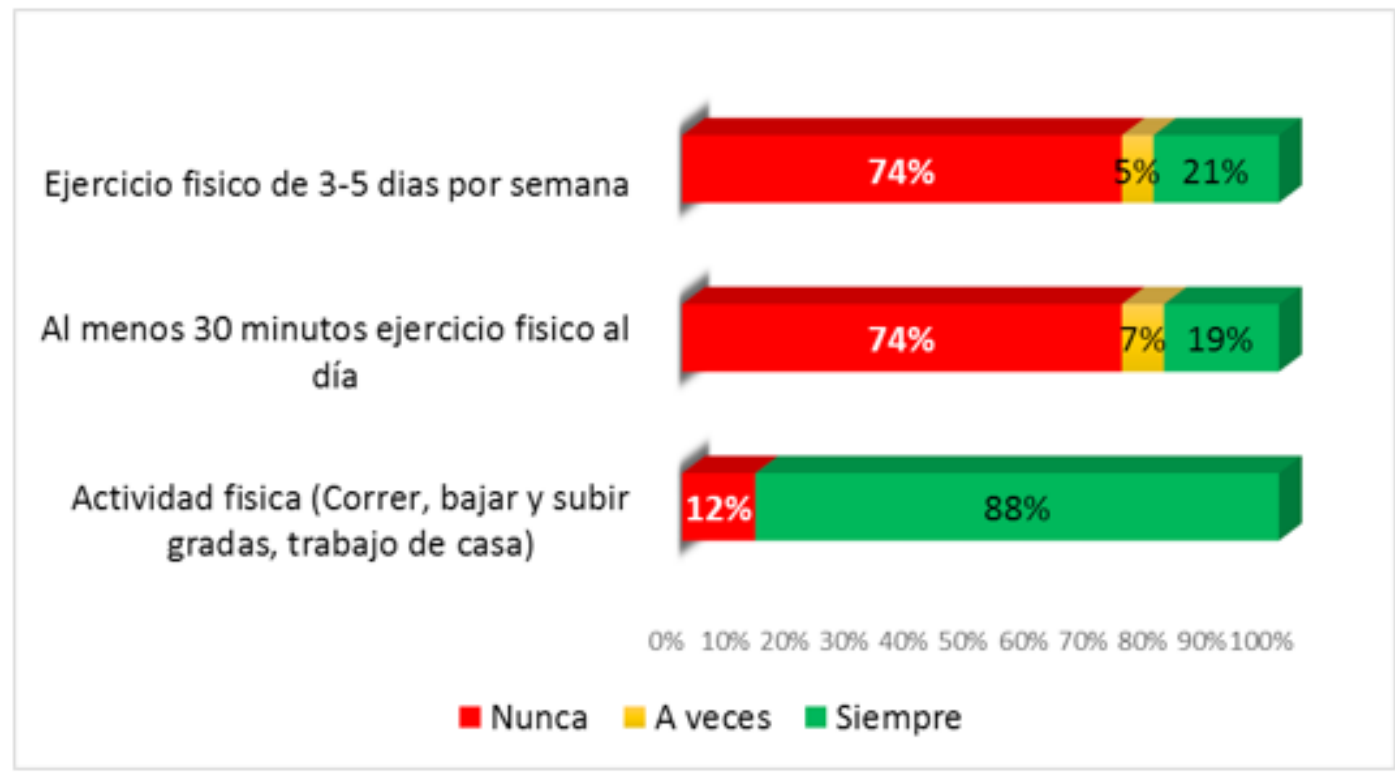

Figura 3. Frecuencia con que usualmente los trabajadores realizan actividades físicas

Los datos para evaluar el comportamiento relacionado al manejo de estrés y horas sueño evidencian que el $70 \%$ de los trabajadores presentan una adecuada práctica, encontrándose el resto con otro factor de riesgo que los está predisponiendo a tener problemas en su peso. Un 
estudio realizado por Durán (2012) sobre la relación entre estado nutricional y sueño concluye que, la restricción de sueño es un factor asociado con la presencia de obesidad en la muestra estudiada.

En cuanto al consumo y uso de sustancias tóxicas, como alcohol y tabaco, únicamente el 16 \% resultó tener una inadecuada práctica, situación que pone en riesgo su salud pues el consumo de alcohol tiene una estrecha relación con enfermedades vasculares y hepáticas, además, provoca mala absorción de nutrientes y debido a su alto contenido energético contribuye a los problemas de peso por exceso, más aún, si esta práctica se acompaña del hábito tabáquico (Carmena, 1999).

La tabla 1 muestra que en la población estudiada y con los datos recopilados, no existe relación estadísticamente significativa entre los estilos de vida y el estado nutricional con un $\boldsymbol{p}=\mathbf{0 . 4 2 0}$, puesto que independientemente de su estilo de vida, más de las tres cuartas partes de los trabajadores se encuentra en sobrepeso u obesidad. Caso similar se observó en el estudio de Torrejón (2012), en el adulto mayor en el que más de la mitad de la muestra presentó un estilo de vida no saludable y el estado nutricional norma l, el cual, tampoco presentó una relación estadísticamente significativa.

Tabla 1. Relación del Estado nutricional de los encuestados y el Estilo de vida

\begin{tabular}{|c|c|c|c|c|c|}
\hline & & & \multicolumn{2}{|c|}{ Estilo de vida } & \multirow[b]{2}{*}{ Total } \\
\hline & & & Inadecuado & Adecuado & \\
\hline \multirow{10}{*}{$\begin{array}{l}\text { Estado nutricional de } \\
\text { los encuestados }\end{array}$} & \multirow{2}{*}{ Normo peso } & Recuento & 5 & 3 & 8 \\
\hline & & $\%$ del total & $8.8 \%$ & $5.3 \%$ & $14.0 \%$ \\
\hline & \multirow{2}{*}{ Sobrepeso } & Recuento & 17 & 15 & 32 \\
\hline & & $\%$ del total & $29.8 \%$ & $26.3 \%$ & $56.1 \%$ \\
\hline & \multirow{2}{*}{ Obesidad I } & Recuento & 3 & 6 & 9 \\
\hline & & $\%$ del total & $5.3 \%$ & $10.5 \%$ & $15.8 \%$ \\
\hline & \multirow{2}{*}{ Obesidad II } & Recuento & 2 & 5 & 7 \\
\hline & & $\%$ del total & $3.5 \%$ & $8.8 \%$ & $12.3 \%$ \\
\hline & Ohorid nd IIU & Recuento & 1 & 0 & 1 \\
\hline & UNesiudu III & $\%$ del total & $1.8 \%$ & $0.0 \%$ & $1.8 \%$ \\
\hline \multirow{4}{*}{ Total } & & Recuento & 28 & 29 & 57 \\
\hline & & $\%$ del total & $49.1 \%$ & $50.9 \%$ & $100.0 \%$ \\
\hline & \multicolumn{5}{|c|}{ Medidas simétricas } \\
\hline & & \multicolumn{2}{|c|}{ Valor } & \multicolumn{2}{|c|}{ Sig. aproximada } \\
\hline \multirow[t]{2}{*}{ Nominal por nominal } & Phi & \multicolumn{2}{|c|}{.261} & \multicolumn{2}{|c|}{.420} \\
\hline & V de Cramer & \multicolumn{2}{|c|}{.261} & \multicolumn{2}{|c|}{.420} \\
\hline $\mathrm{N}$ de casos válidos & \multicolumn{3}{|c|}{57} & & \\
\hline
\end{tabular}




\section{CONCLUSIÓN}

Este estudio muestra datos que antes no se habían investigado en una población de trabajadores docentes y administrativos de una unidad de estudios superiores que forma profesionales afines con la salud. La presente investigación ha permitido obtener las siguientes conclusiones:

a. En cuanto a las condiciones sociodemográficas, más de la mitad de los trabajadores se encuentran dentro de las edades de 21 a 48 años; el sexo que predominó fue el femenino y en su mayoría pertenecen al casco urbano.

b. Más de tres partes de los trabajadores se encontraron con sobrepeso o algún grado de obesidad. Los docentes salieron más afectados que los administrativos. En cuanto a esta condición de salud y los que se encuentran normales, no presentan ninguna patología asociada.

c. Dentro de los hábitos alimentarios demuestran prácticas inadecuadas como: agregar sal a los alimentos y consumir refrescos industrializados. El patrón de consumo lo componen 10 alimentos mostrando una dieta poco diversa. La fuente principal de adquisición calórica son las grasas y las proteínas encontrando un desbalance en la adecuación calórica. Casi tres partes de los trabajadores no practican ningún tipo de ejercicio programado, presentan un buen manejo del tiempo libre y estrés, poco consumo de alcohol y tabaco.

d. De acuerdo al análisis estadístico inferencial en este caso y con estos datos, no existe una relación estadísticamente significativa entre los estilos de vida y el estado nutricional de los trabajadores del POLISAL.

\section{REFERENCIAS BIBLIOGRÁFICAS}

Aburto, A. (2006). Metodología del estudio del patrón alimentario. Nicaragua.

Aranceta, J. P. (2005). Prevalencia de obesidad en españa. Medicina clínica, 125(12), 608-

Bracker, M. (Octubre de 1998). Mòdulo " La entrevista cualitativa " Tomo I. Managua, Nicaragua.

Carmena, R. R. (1999). Dieta, lìpidos y arterosclerosis. En R. M. Hernández,
Tratado de Nutrición (pág. 1041). Madrid: Editorial Díaz de Santos.

Durán S., F. N. (marzo de 2012). Relación entre estado nutricional y sueño en escolares de la comuna de San Miguel, Santiago, Chile. Revista chilena de nutrición, 39(1), 30-37.
FAO. (2007). Guía de seguridad alimentaria y nutricional para uso del personal agropecuario Nicaragua. Obtenido de https://coin.fao.org/coin-static/cms/ 
media/13/13436723079830/guia_de_ seguridad_alimentaria_y_nutricional.pdf

FAO. (2014). Obtenido de http://www.fao. org/docrep/019/i3520s/i3520s.pdf

FAO. (s.f.). Disponibilidad de alimentos para la soberania y seguridad alimentaria. FAO.

FUNIDES. (23 de 01 de 2012). www.funides. com/documentos/.../primer_informe_ coyuntura_2013. Recuperado el 15 de 07 de 2014

González, M. P. (15 de Noviembre de 2009). SciELO. Obtenido de http:// scielo.isciii.es/scielo.php?pid=S0212$16112011000200015 \& \mathrm{script}=\mathrm{sci}$ arttext\&tlng=en

OMS. (2010). Obtenido de http:// www.who.int/dietphysicalactivity/ publications/9789241599979/es/

OMS. (2011). Organización Mundial de la Salud. Recuperado el 17 de Julio de 2014, de www.who.int/topics/chronic_diseases/ es/

Palomares, L. (2014). Recuperado el 2017, de http://repositorioacademico.upc.edu. pe/upc/bitstream/10757/566985/2/ TesisdeMaestr\%C3\%ADa_LitaPalomares. pdf
Pastor, Y. (1998). Dimenciones del estilo de vida relacionados con la salud en la adolescencia: una revisión. Obtenido de Dialnet: https://dialnet.unirioja.es/descarga/ articulo/2498026.pdf

Ratner, R. S. (2008). Revista Médica. Obtenido de http://repositorio.uchile.cl/ handle/2250/128177

Salinas, J. B. (2005). Actividad física integral con adultos y adultos mayores en Chile: resultados de un programa piloto. Obtenido de http:// www.scielo.cl/scielo.php?pid=S071775182005000300006\&script=sci_arttext

Sanabria Ferrand, P. A., Gonzalez, L. \& Urrego, M. D. (2007). Estilos de vida saludable en profesionales de la salud colombianos. Estudio exploratorio. Med, 207-217. Obtenido de http://www.redalyc.org/ articulo.oa?id=91015208

Torrejón, C. \& Márquez, R. (2012). Obtenido de http://revistas.uladech.edu.pe/index. php/increscendo/article/view/116 